\title{
Home therapy for inherited bleeding disorders in South Africa: Results of a modified Delphi consensus process
}

\author{
J N Mahlangu, ${ }^{1} \mathrm{MB}$ BCh, FC Path (SA), MMed (Haem), Cert Clinical Haematology (SA); \\ Y Naidoo, ${ }^{2} \mathrm{MB}$ ChB, FC Path (SA), Cert Clinical Haematology (SA); Y Goga, ${ }^{3} \mathrm{MB}$ ChB, FC Paed (SA); \\ M Vaithlingum, ${ }^{4}$ MB ChB, FC Paed (SA), Cert Clinical Haematology (SA); J Joubert, ${ }^{5}$ MB ChB, MMed (Haem), PG Dip; \\ C Sutton, ${ }^{6} \mathrm{MB}$ BCh, FC Paed (SA); J Potgieter, ${ }^{7} \mathrm{MB}$ ChB, MMed (Haem); A-L Cruickshank, ${ }^{8} \mathrm{RN}, \mathrm{RM}$; A Iorio, ${ }^{9} \mathrm{MD}, \mathrm{FRCPC}, \mathrm{PhD}$ \\ ${ }^{1}$ Haemophilia Comprehensive Centre, Charlotte Maxeke Academic Johannesburg Hospital, National Health Laboratory Service and
University of the Witwatersrand, Johannesburg, South Africa
${ }^{2}$ Lilly Haematology Centre, Midlands Medical Centre, KwaZulu-Natal, South Africa
${ }^{3}$ Inkosi Albert Luthuli Central Hospital and Department of Paediatrics, Nelson Mandela School of Medicine, College of Health Sciences,
University of KwaZulu-Natal, Durban, South Africa
${ }^{4}$ Netcare Parklands Hospital, Durban, South Africa
${ }^{5}$ Division of Clinical Haematology, Faculty of Health Sciences, University of the Free State, Bloemfontein, South Africa
${ }^{6}$ Department of Paediatrics, Faculty of Health Sciences, University of Limpopo, South Africa
${ }^{7}$ Steve Biko Academic Hospital and Department of Haematology, Faculty of Health Sciences, University of Pretoria and National Health
Laboratory Service, South Africa
${ }^{8}$ Groote Schuur Hospital and Department of Haematology, Faculty of Health Sciences, University of Cape Town, South Africa
${ }^{9}$ Department of Health Research, Impact and Evidence and Department of Medicine, McMaster University, Hamilton, Ontario, Canada
}

Corresponding author: J N Mahlangu (johnny.mahlangu@nhls.ac.za)

Background. Optimal care of patients with inherited bleeding disorders requires that bleeding episodes are treated early, or still better prevented, through extension of patient care beyond hospital-based treatment to home-based therapy. In South Africa (SA), adoption of home therapy is variable, in part owing to lack of consensus among healthcare providers on what constitutes home therapy, which patients should be candidates for it, how it should be monitored, and what the barriers to home therapy are.

Objectives. To conduct a modified Delphi process in order to establish consensus on home therapy among haemophilia healthcare providers in SA.

Methods. Treaters experienced in haemophilia care were invited to participate in a consensus-seeking process conducted in three rounds. In round 1, provisional statements around home therapy were formulated as questions and collated in a structured list. In rounds 2 and 3 , evolving versions of the questionnaire were administered to participants. Consensus was defined as $\geq 70 \%$ agreement among the participants. Results. The panel composition included an equal number of physicians and non-physicians. The participation rate was $100 \%$ through all three consensus rounds. The group reached consensus for $92 \%$ of the statements. Consensus of $100 \%$ was reached on starting home therapy in paediatric patients, requiring all patients on home therapy to sign informed consent and indemnity, and providing round-the-clock support for patients on home therapy.

Conclusions. The home therapy consensus statements in this report have the potential to translate to policy on home therapy and to guide the initiation, practice and evaluation of home therapy programmes in SA.

S Afr Med J 2019;109(9):639-644. https://doi.org/10.7196/SAMJ.2019.v109i9.13637

Inherited bleeding disorders are heterogeneous in aetiology and clinical presentation and may arise from platelet, blood vessel or clotting factor structural or functional abnormalities. ${ }^{[1]}$ Haemophilia is the most common X-linked condition, affecting 1 in 5000 and 1 in 25000 live male births for haemophilia A and B, respectively. ${ }^{[2]}$ In South Africa (SA), with a population of 57700 million people, ${ }^{[3]}$ the expected number of people with haemophilia is 5770 , of whom only $\sim 200$ have been diagnosed. ${ }^{[4]}$ Once diagnosed, haemophilia imposes a high disease and treatment burden on patients, caregivers, healthcare providers and the healthcare facilities involved in management. The disease burden includes unpredictable spontaneous acute bleeds as well as chronic complications of bleeds into the musculoskeletal system, muscle and soft tissues, which may be life or organ threatening or disabling. ${ }^{[5-8]}$ The treatment burden is in part due to lifelong treatment for spontaneous and traumatic bleed ${ }^{[9,10]}$ with clotting factor concentrate (CFC) replacement therapy, administered by intravenous infusion and therefore requiring specific care processes and skills.

Current management of bleeds in haemophilia is by intravenous replacement of the deficient clotting factor to treat or prevent bleeding episodes. ${ }^{[1]}$ Episodic treatment, the reactive modality, is associated with progressive deterioration of musculoskeletal structures, musculoskeletal function and quality of life. ${ }^{[12]}$ The proactive alternative is prophylaxis, the standard of care in haemophilia, in which patients receive regular infusions of clotting factor to prevent bleed occurrence. ${ }^{[13,14]}$ Replacement therapy, whether given episodically or prophylactically, may be delayed while patients are transported to healthcare facilities. This delay has been shown to result in complications of bleeds, which become more difficult to manage, delayed in their resolution, and associated with 
poor musculoskeletal and quality-of-life outcomes. ${ }^{[15-17]}$ The delay in intravenous therapy can be mitigated by teaching patients how to practise intravenous infusion in their home settings. Indeed, in many comprehensive treatment centres in SA, patients are taught selfinfusion at an early age. ${ }^{[4]}$ It has now been shown in many studies that intravenous infusion skill combined with early replacement therapy has the potential to limit bleed-induced musculoskeletal damage, limit the extent of blood loss, and improve bleed resolution and quality of life of haemophilia patients. ${ }^{[18-21]}$ Recent data also indicate that early home-based therapy is cost-effective, and is associated with reduced pain and disability, decreased school and work absenteeism, and reduced hospitalisation rates. ${ }^{[22-24]}$ Consequently, in many parts of the world, haemophilia patients are allowed to take CFC at home in order to infuse themselves prophylactically or to self-administer early during an acute bleeding episode.

In a survey performed in 10 haemophilia treatment centres in SA, the practice of home therapy varied from $0 \%$ to $70 \%$. Part of the explanation for this wide variability in practice is that there was no consensus among the healthcare providers on a number of questions on home therapy-related issues for patients with inherited bleeding disorders. The unanswered questions include: (i) who should be allowed to take CFC home in order to self-infuse; (ii) what the barriers to home therapy would be; (iii) what the criteria for identifying patients for home therapy should be; (iv) what the characteristics of products for use in the home setting should be; $(v)$ what kind of support would be required for patients on home therapy; and ( $v i$ ) how the effectiveness of home therapy should be monitored.

The Delphi approach is an established method for consensus building by using a series of reiterated questionnaires to facilitate a panel of selected experts to reach agreement on a particular subject. ${ }^{[25,26]}$ The Delphi approach differs from standard surveys by asking the question as what should/would be, as opposed to surveys, which ask the question as what is. ${ }^{[27]}$ It is particularly appropriate to use the Delphi method in rare diseases such as haemophilia for which prospectively generated data might take time or be difficult to collect, and particularly for questions that would be impractical to answer via other forms of research. ${ }^{[28]}$ The Delphi method has frequently been used in haemophilia to reach expert consensus on a variety of issues. ${ }^{[29-32]}$

\section{Objectives}

This study used a modified Delphi method to seek consensus among haemophilia experts on key factors required for a successful home therapy programme for patients with inherited bleeding disorders in SA.

\section{Methods}

\section{Study participants}

Invited participants had expertise and knowledge in the care of patients with haemophilia and included medical practitioners, nurse practitioners and allied healthcare workers practising in haemophilia. They were representative of the haemophilia fraternity in SA in terms of expertise, experience, haemophilia population they cared for, and healthcare professions.

\section{Study design and interventions}

The Delphi method was administered by an independent facilitator in three rounds.

\section{Round 1}

In a face-to-face meeting, the objective and procedures were outlined to all participants. Participants were assigned to two groups and tasked to identify all haemophilia patient-, home setting-, healthcare provider-, treatment centre- and healthcare system-related factors involved in implementation of a successful home therapy programme. Participants considered each factor identified and the rationale for its inclusion. They were not allowed to give individual opinions, recommendations or consensus at this stage. All inputs were examined by the whole group in order to clarify possible ambiguous statements and remove duplicates.

The resultant list of issues on haemophilia home therapy was then formulated into questions, which were captured in a study questionnaire. The questionnaire was checked by a small core group of participants for completeness, consistency and clarity before being finalised. Care was taken that the statements were not rated or ranked at this stage.

\section{Round 2}

The finalised questionnaire was distributed to all participants through an online tool provided by McMaster University in Canada. Each participant received an invitation to complete the online questionnaire individually. Participants were not allowed to confer or discuss their input with each other. The timeline for completion of the survey was a maximum of 5 days after the first notification. At the end of 5 days, all responses were centrally collated, analysed and interpreted.

\section{Round 3}

Consensus statements from round 2 were used to proceed to round 3. All participants were asked to rank the statements in order of importance. The results were collated and checked for the level of agreement or disagreement among the participants.

Consensus was defined as $\geq 70 \%$ of the participants agreeing or disagreeing on a statement. Once consensus was reached, the statements were ranked from most to least important based on the rank received. The participant responses were collated, analysed and interpreted.

\section{Results}

\section{Participant demographics}

There were 20 participants who took part in the first meeting of the group, and the same number remained throughout the entire consensus process without dropouts. The demographic profile of the study participants is shown in Table 1. Our participating healthcare providers were all experienced in haemophilia care, with 18 of the 20 having $\geq 6$ years of working in haemophilia care.

\section{Participant response rate and consensus}

The participant response rate in round 2 and round 3 was 100\%, reflecting the commitment of this group to the process. In round 2, consensus was reached in 33 of the 36 questions (92\%).

The top three statements for which there was $100 \%$ expert agreement are listed in Table 2. The three statements for which there was no agreement among the study participants are listed in Table 3. There was general agreement for the remainder of the questionnaire statements, which are listed in Table 4. The consensus group identified several potential barriers to home therapy, and the top 10 of these are listed in Table 5.

\section{Discussion}

Our study participants all agreed that home therapy should target the paediatric population as a priority over the adult population, that patients on home therapy should sign an informed consent and an indemnity, and that patients on home therapy should have roundthe-clock access to a healthcare provider (Table 2). 
As indicated in Table 1, a high proportion of participants in the group looked after paediatric patients, which may have skewed the consensus in favour of children. However, there were sufficient nonpaediatric participants to have changed this if there was no consensus. The rationale for targeting the paediatric group is the impact home therapy will have in preventing and reducing the haemophilia disease burden in this age group. Owing to poor venous access, many home therapy guidelines do not specifically recommend targeting the paediatric group for home therapy. ${ }^{[33-35]}$ In a resource-constrained setting such as ours, our group considered the cost-effectiveness of home therapy in terms of volume of CFC to keep at home, as well as the long distances our patients have to travel to reach haemophilia treatment centres. In general, paediatric patients require fewer vials of CFC at home, and more than 10 of the 18 haemophilia treatment centres in SA are servicing patients drawn from rural settings.

The recommendation that patients on home therapy should sign an informed consent and an indemnity came as no surprise. SA is not a litigious society; however, we have seen a steep rise in healthrelated litigations, particularly in state facilities where patients claim professional negligence. ${ }^{[36]}$ This recommendation is also in line with increasing societal demands for the practice of ethical medicine in SA and aligns with current hospital practice for patients undergoing

\begin{tabular}{|c|c|}
\hline & $n$ \\
\hline Number of participants & 20 \\
\hline \multicolumn{2}{|l|}{ Participant professions } \\
\hline Doctor & 10 \\
\hline Nurse & 9 \\
\hline Physiotherapist & 1 \\
\hline \multicolumn{2}{|c|}{ Experience in haemophilia care (years) } \\
\hline$\leq 5$ & 2 \\
\hline $6-10$ & 6 \\
\hline $11-15$ & 4 \\
\hline $16-20$ & 4 \\
\hline$>20$ & 4 \\
\hline \multicolumn{2}{|l|}{ Type of practice } \\
\hline Adult haemophilia only & 3 \\
\hline Paediatric haemophilia only & 7 \\
\hline Combined adult/paediatric & 10 \\
\hline \multicolumn{2}{|c|}{ Number of haemophilia patients in the practice } \\
\hline $20-100$ & 12 \\
\hline $101-200$ & 2 \\
\hline $201-300$ & 1 \\
\hline $301-400$ & 1 \\
\hline $401-500$ & 2 \\
\hline$>500$ & 2 \\
\hline
\end{tabular}

procedures to sign indemnity for hospitalisation and consent for all procedures. Once implemented, this recommendation will give patients and healthcare providers the reassurance that home therapy is taken as seriously as any other procedures performed in healthcare facilities.

The above fact links to the third top recommendation, that patients on home therapy should have round-the-clock access to a healthcare provider. Such support is already provided for all haemophilia patients, but becomes mandatory for patients who will be infusing $\mathrm{CFC}$ at home, in view of potential complications of poor venous access.

The participant experts in this Delphi process comprised 10 doctors, 9 nurses and a physiotherapist. The equal number of doctor and non-doctor participants in this expert sample reflects the kind of collective decision-making that takes place in the care of patients with haemophilia. While other members of the haemophilia comprehensive care team were considered for inclusion, these panel members were thought to be pivotal in the direct management of haemophilia patients on home therapy. Unlike other Delphi processes in haemophilia, which have often excluded nurses, ${ }^{[29,32]}$ our process included nurses, as they are critical role players in the instruction of patients on home therapy as well as in their follow-up and treatment monitoring.

Our participating healthcare providers were all experienced in haemophilia care, with 18 of the 20 having $\geq 6$ years of working in haemophilia care. This is important, as the decisions taken on home therapy have lifelong implications for patients and their caregivers and therefore require considerable experience and knowledge in the haemophilia field. Our Delphi participants did not include administrators or patients, as healthcare providers wanted to reach consensus among themselves before including patients and administrators in the medical decision-making.

The Delphi process participants did not reach consensus on 3 of the 36 statements in the questionnaire. The reasons for failing to reach consensus are fairly clear and reflect the influence of the composition of the group and the age and size of patients they look after. With regard to needle size, the non-consensus ultimately became a disagreement between paediatricians and adult treaters. In the final analysis, this disagreement was an academic exercise, as all CFC comes with pre-packed needles of the same size. On the home environment, paediatricians felt strongly that only knowledgeable, motivated and skilled caregivers should be allowed to participate in the home therapy programme. How much CFC patients take home is ultimately at the discretion of each hospital, and their policy is often informed by whether the patient is on episodic or prophylactic treatment. The generally accepted principle is for patients to have enough CFC to treat at least one bleed at home or to have enough CFC not to miss prophylactic doses until the next scheduled treatment centre visit. Current national and international guidelines are silent on these two aspects. ${ }^{[37,38]}$

\section{Table 2. Top three questionnaire statements for which there was $100 \%$ agreement among participants}

- Treatment centres should prioritise paediatric patients for home therapy

- Patients on home therapy should sign informed consent and indemnity for participating in the home therapy programme

- Patients on home therapy should be provided with 24 -hour round-the-clock support by the treatment centre

- Sizes of needles supplied to people with haemophilia for home therapy

- Number of doses to be dispensed for home therapy 
Table 4. Statements of consensus among participants ( $>70 \%$ agreement per statement)

\section{Questionnaire statement}

1. Definition of home therapy

2. Goals and rationale for implementing home therapy need to be defined

3. Age of starting home therapy

4. Factors to consider before starting home therapy

5. Patient characteristics that should favour home therapy

6. Issues of venous access in the home setting

7. Success indicators for home therapy

8. Patients needing particular attention in the home therapy programme

9. Important role players required for the support patients on home therapy

10. Characteristics of haemophilia centres practising home therapy

11. Characteristics of products used in the home therapy programmes

12. Factors to consider when monitoring home therapy
Agreement reached

- Home therapy is therapy taken by haemophilia patients at home under supervision of the haemophilia care team

- The primary goals of home therapy should be to treat bleeds early or to put patients on prophylaxis in order to reduce bleed rates and associated musculoskeletal complications

- Home therapy should be started as soon as the caregivers and patients have been instructed on intravenous infusion and deemed competent

- Having a fridge to store product at home

- Supportive and nurturing home setting

- Hygiene conditions at home

- Privacy at home

- Distance of home from hospital

- Ability to contact the treatment team

- Patients should be competent in IV needle insertion

- Patients should be reliable and trustworthy

- Patients should be able to identify the complications of IV needle insertion

- Patients should understand the importance of a sterile field when inserting the IV needle

- Patients should be able to dispose of needles safely

- Patients must have passed at least one course on home therapy

- Central venous and arterial lines should be avoided

- A maximum of three unsuccessful venous stabs should require expert intervention

- Should include evaluation of annualised bleeding rate

- Number of missed injections for patients on prophylaxis

- Serial measurement of musculoskeletal haemophilia complications

- Number of deaths of patients on home therapy

- Improvement in the quality of life of patients

- Poor venous access

- Inhibitor patients requiring bypassing agents

- Poor home support structure

- Non-compliant patients

- Patients who have failed home therapy training

- Patients unable to handle and store clotting factor concentrate

- Immediate parent/guardian/spouse

- Extended family

- Friends and neighbours

- Teachers and co-workers

- Local clinic sisters

- Emergency personnel

- Ability to supply unlimited quantities of factor

- Availability of staff 24 hours a day to support patients

- Adequate treatment centre drug stock level

- Ability to monitor and review treatment compliance

- Ability to monitor home therapy impact and outcomes

- Ability of the treatment centre to train and monitor patients

- The product should be non-thrombotic

- The product should be non-immunogenic in previously untreated patients

- The product should not be associated with allergy or anaphylaxis

- The product should not cause local reaction on skin extravasation

- Product overdose should be very well tolerated

- Volume of product consumption and accountability

- Number of bleeds in a patient on prophylaxis

- Number of doses required to treat a bleeding episode

- Number of times the patient has run out of medication

- Number of calls the patient makes to the healthcare providers 
Table 5. Potential barriers to home therapy identified by the participants

- Inadequate budgets in health facilities for home therapy

- Healthcare providers rationing available clotting factor replacement therapy for patients

- Lack of adequate clotting factors in health facilities

- Lack of appropriate protocols and tools to initiate, implement, monitor and evaluate home therapy

- Poor evaluation and monitoring of home therapy outcomes by healthcare providers

- Generally non-compliant patients

- Patients with poor venous access

- Patients who have failed home therapy training

- Patients with poor home support structure

- Patient unable to handle and store clotting factor appropriately

There was non-unanimous but significant consensus among the Delphi group members on the remaining statements listed in Table 4. These statements will have the potential for informing policymaking and becoming part of standard operating procedures for all treatment centres in SA. The standardisation of procedures and policies will help reduce the current largely discrepant practices of home therapy healthcare providers.

To our knowledge, this study is the first to examine home therapy closely and to seek consensus among haemophilia healthcare providers in the developing world. The reasons for lack of similar studies in the past remain unclear, but could include the fact that expertise in haemophilia in many resource-constrained settings is limited and the very practice of home therapy might be poorly supported. In contrast, in many developed countries home therapy is regarded as part of the standard of care of haemophilia, and the need for consensus to implement it therefore does not exist.

The recommendations on home therapy would have been incomplete without identifying potential barriers and hurdles to its implementation. The biggest barrier identified from this Delphi process was the healthcare provider being the resource gatekeeper and not allowing patients to self-infuse. We managed to keep our Delphi process free of interference from the healthcare facility administrators, yet there were a number of haemophilia treatment centres in which patients who were adequately trained on home therapy were not allowed to take CFC to infuse themselves at home by either their healthcare team or hospital administrators. It is hoped that raising awareness around this practice will result in rational decision-making and alignment of the dispensing practice with the recommendations of this study.

\section{Study strengths and limitations}

The study strengths included the cohesiveness of the group, illustrated by the fact that none of the participants were lost during the study. The fact that consensus within the group was reached quickly within two iterations indicated the general convergence of thinking within the group, which it is hoped will translate to uniform policy and practice in SA.

The study had some limitations. The participants were self-selected and therefore may not be representative of haemophilia healthcare providers as a whole in SA. They included only a small number of healthcare providers and did not include patients or administrators, who would have provided a non-healthcare provider perspective to the consensus statements.

\section{Conclusions}

Through a modified Delphi process, we have been able to answer a number of questions and reached consensus on haemophilia home therapy, including who should be prioritised for home therapy, the prerequisites for home therapy, and barriers to a successful home therapy programme. The adoption of home therapy in haemophilia will result in a number of desirable outcomes that include reduction in bleeding rates, reduction in musculoskeletal complication rates and improvement in quality of life. ${ }^{[39-41]}$ The consensus statements arising from this Delphi process will serve as a foundation for formulating a home therapy national policy and for narrowing the wide home therapy practice gap currently seen among the haemophilia treatment centres in SA.

\section{Declaration. None.}

Acknowledgements. The authors thank the following individuals who participated in the consensus process and/or meetings: Emma Iserman, McMaster University; David Stones, University of the Free State; Rajendra Thejpal, University of KwaZulu-Natal; Sameer Rahim, Red Cross War Memorial Children's Hospital; Sibongile Mbhele, Charlotte Maxeke Johannesburg Hospital; Alice Banze, Haemophilia Foundation of South Africa; Nombuyiselo Thuswa, Haemophilia Foundation of South Africa; Rosemary Khanyile, Nelson Mandela Academic Hospital; Nadine Rapiti, Inkosi Albert Luthuli Central Hospital; Nomawethu Tonjeni, Nelson Mandela Academic Hospital; and Beverley Neethling, Grey’s Hospital. Author contributions. JNM convened the group, formulated the questionnaire, participated in the consensus process, collated responses, wrote the manuscript, and critically reviewed and edited the manuscript. JP conceptualised the idea and obtained support from Novo Nordisk, participated in the consensus process, and critically reviewed and edited the manuscript. YN participated in the consensus process, chaired the feedback meeting, and critically reviewed and edited the manuscript. YG participated in the consensus process, and critically reviewed and edited the manuscript. MV participated in the consensus process, and critically reviewed and edited the manuscript. JJ participated in the feedback meeting, and critically reviewed and edited the manuscript. CS participated in the consensus process, and critically reviewed and edited the manuscript. A-LC participated in the consensus process, and critically reviewed and edited the manuscript. AI provided methodological support in setting up and running the Delphi process, performed data analysis, and critically reviewed and edited the manuscript.

Funding. The cost of participation in the face-to-face meeting was funded by an unrestricted educational grant from Novo Nordisk South Africa. Novo Nordisk did not have any input into the collection, analysis, interpretation or reporting of this study.

Conflicts of interest. None.

1. Lippi G, Franchini M, Guidi GC. Diagnostic approach to inherited bleeding disorders. Clin Chem Lab Med 2007;45(1):2-12. https://doi.org/10.1515/CCLM.2007.006 
2. Srivastava A, Brewer AK, Mauser-Bunschoten EP, et al. Guidelines for the management of hemophilia. Hemophilia 2012;19(1):el-e47. https://doi.org/10.1111/j.1365-2516.2012.02909.x

3. Statistics South Africa. Update of the South African population. http://www.statssa.gov.za/?p=11341 (accessed 12 August 2018)

4. Mahlangu JN. Haemophilia care in South Africa: 2004 - 2007 look back. Hemophilia 2008:15(1):135 141. https://doi.org/10.1111/j.1365-2516.2008.01807

5. Price VE, Hawes SA, Bouchard A, et al. Unmeasured costs of haemophilia: The economic burden on families with children with haemophilia. Haemophilia 2015;21(4):e294-e299. https://doi.org/10.1111/ hae. 12715

6. Lindvall K, von Mackensen S, Elmstahl S, et al. Increased burden on caregivers of having a child with haemophilia complicated by inhibitors. Pediatr Blood Cancer 2014;61(4):706-711. https://doi. org/10.1002/pbc.24856

7. Gringeri A, Ewenstein B, Reininger A. The burden of bleeding in haemophilia: Is one bleed too many? Haemophilia 2014;20(4):459-463. https://doi.org/10.1111/hae.12375

8. Gater A, Thomson TA, Strandberg-Larsen M. Haemophilia B: Impact on patients and economic burden of disease. Thromb Haemost 2011;106(3):398-404. https://doi.org/10.1160/TH11-03-0193

9. McMullen S, Buckley B, Hall E 2nd, et al. Budget impact analysis of prolonged half-life recombinant FVIII therapy for hemophilia in the United States. Value Health 2017;20(1):93-99. https:/doi. org/10.1016/j.jval.2016.09.2396

10. Frampton JE. Efmoroctocog alfa: A review in haemophilia A. Drugs 2016;76(13):1281-1291. https:// doi.org/10.1007/s40265-016-0622-z

11. Srivastava A, Brewer AK, Mauser-Bunschoten EP, et al. Guidelines for the management of hemophilia. Haemophilia 2013;19(1):e1-e47. https://doi.org/10.1111/j.1365-2516.2012.02909

12. Poonnoose P, Carneiro JDA, Cruickshank AL, et al. Episodic replacement of clotting factor concentrates does not prevent bleeding or musculoskeletal damage - the MUSFIH study. Haemophilia 2017;23(4):538-546. https./1doi

3. Makris M. Prophylaxis in haemophilia should be life-long. Blood Transfus 2012;10:165-168. https:// doi.org/10.2450/2012.0147-11

4. Franchini M, Mannucci PM. Prophylaxis for adults with haemophilia: Towards a personalised approach? Blood Transfus 2012;10:123-124. https://doi.org/10.2450/2012.0182-1

15. Van den Berg HM. Preventing bleeds by treatment: New era for haemophilia changing the paradigm. Haemophilia 2016;22(Suppl 5):9-13. https://doi.org/10.1111/hae.12993

16. Feltmate K, Janiszewski PM, Gingerich S, et al. Delayed access to treatments for rare diseases: Who's to blame? Respirology 2015;20(3):361-369. https://doi.org/10.1111/resp.12498

17. Valentino LA, Walsh CE, Reding MT, et al. Patient- and caregiver-reported bleeding symptoms and reasons for starting and stopping treatment with recombinant factor VIIa: Analysis of the Dosing Observational Study in Haemophilia (DOSE). Haemophilia 2012;18(4):554-560. https://doi. Dosing Observational Study in Haem

18. Oldenburg J, Austin SK, Kessler CM. ITI choice for the optimal management of inhibitor patients from a clinical and pharmacoeconomic perspective. Haemophilia 2014;20(Suppl 6):17-26. https://doi. from a clinical and phar

19. Oyesiku JO. Home treatment of haemophilia patients with inhibitors. Haemophilia 2011;17(2):173178. https://doi.org/10.1111/j.1365-2516.2010.02418

20. Kavakli K, Yesilipek A, Antmen B, et al. The value of early treatment in patients with haemophilia and inhibitors. Haemophilia 2010;16(3):487-494. https://doi.org/10.1111/j.1365-2516.2009.02179.x

21. Holme PA, Glomstein A, Gronhaug S, et al. Home treatment with bypassing products in inhibito patients: A 7.5-year experience. Haemophilia 2009;15(3):727-732. https://doi.org/10.1111/j.1365 2516.2009.02017.x

22. Henry N, Jovanovic J, Schlueter M, et al. Cost-utility analysis of life-long prophylaxis with recombinant factor VIIIFc vs recombinant factor VIII for the management of severe hemophilia A in Sweden. J Med Econ 2018:21(4):318-325. https://doi.org/10.1080/136969982017.1405816
23. Hay JW, Zhou ZY. Systematic literature review of economics analysis on treatment of mild-to-moderate bleeds with aPCC versus rFVIIa. J Med Econ 2011;14(4):516-525. https://doi.org/10.3111/13696998.2 011.595462

24. Knight $C$. Dano AM, Kennedy-Martin T. A systematic review of the cost-effectiveness of $r F V I I a$ and APCC in the treatment of minor/moderate bleeding episodes for haemophilia patients with inhibitors. Haemophilia 2009;15(2):405-419. https://doi.org/10.1111/j.1365-2516.2008.01969.x

25. Rand Corporation. Delphi method. https://www.rand.org/topics/delphi-method.html (accessed 13 August 2018).

26. Dalkey NC, Helmer O. An experimental application of the Delphi method to the use of experts. Manag Sci 1963;9:458-467.

27. HSU C-C. The Delphi Technique: Making sense of consensus. Practical Assessment, Research and Evaluation 2007;12(10):1-8.

28. Mullen PM. Delphi: Myths and reality. J Health Organ Manag 2003;17(1):37-52. https://doi. org/10.1108/14777260310469319

29. McCarthy A, Moore A, Redhead L, et al. Development of haemophilic arthropathy of the ankle: Results of a Delphi consensus survey on potential contributory factors. Haemophilia 2015;21(1):116-123. https:// doi.org/10.1111/hae. 12500

30. Linertova R, Serrano-Aguilar P, Posada-de-la-Paz M, et al. Delphi approach to select rare diseases for a European representative survey: The BURQOL-RD study. Health Policy 2012;108(1):19-26. https://doi, rg/10.1016/j.healthpol.2012.08.001

31. Matino D, Lillicrap D, Astermark J, et al. Switching clotting factor concentrates: Considerations in estimating the risk of immunogenicity. Haemophilia 2014;20(2):200-206. https://doi.org/10.1111/ hae. 12283

32. Barnes C, Brown SA, Curtin J, et al. When is enough ... enough? Developing consensus of definition of failure of immune tolerance induction in patients with haemophilia and inhibitors. Haemophilia 2014;20(4):e275-e279. https://doi.org/10.1111/hae.12442

33. Soucie JM, Symons $J$ 4th, Evatt B, et al. Home-based factor infusion therapy and hospitalization for bleeding complications among males with haemophilia. Haemophilia 2001;7(2):198-206.

34. Jones P. Haemophilia home therapy. Haemostasis 1992;22(5):247-250.

35. Editorial. Haemophilia and home therapy. Lancet 1979;2(8133):77-78. https://doi.org/10.1016/S01406736(79)90125-9

36. health24. Medical malpractice litigation: Undermining South Africa's health system. https://www. health24.com/News/Public-Health/medical-malpractice-litigation-undermining-south-africas-healthsystem-20180417 (accessed 18 October 2018).

37. Srivastava A, Brewer AK, Mauser-Bunschoten EP, et al. Guidelines for the management of hemophilia. Haemophilia 2013;19(1):el-e47. https://doi.org/10.1111/j.1365-2516.2012.02909.X

38. Mahlangu JN, Gilham A. Guideline for the treatment of haemophilia in South Africa. S Afr Med J 2008;98(2):126-140

39. Iorio A, Krishnan S, Myren KJ, et al. Indirect comparisons of efficacy and weekly factor consumption during continuous prophylaxis with recombinant factor VIII $\mathrm{Fc}$ fusion protein and conventional recombinant factor VIII products. Haemophilia 2017;23(3):408-416. https://doi.org/10.1111/hae.13160

0. Berntorp E, Dolan G, Hay C, et al. European retrospective study of real-life haemophilia treatment. Haemophilia 2017;23(1):105-114. https://doi.org/10.1111/hae.13111

41. Chowdary P, Fosbury E, Riddell A, et al. Therapeutic and routine prophylactic properties of rFactor VIII Fc (efraloctocog alfa, Eloctate ${ }^{\circ}$ ) in hemophilia A. J Blood Med 2016;2016(7):187-198. https://doi. org/10.2147/JBM.S8081

Accepted 11 February 2019 